\title{
EVOLUCIÓN Y DESFRAGMENTACIÓN DEL PAISAJE DEL VIÑEDO EN LA RIOJAALTA (ESPAÑA) EN EL PERIODO 1956-2000
}

\author{
Ianire Galilea ${ }^{1}$ \\ José Arnáez ${ }^{1^{*}}$ \\ Teodoro Lasanta², \\ Luis Ortigosa ${ }^{1(t)}$ \\ 'Área de Geografía Física. Universidad de La Rioja \\ *jose.arnaez@unirioja.es \\ ${ }^{2}$ Instituto Pirenaico de Ecología (CSIC). Zaragoza
}

\section{RESUMEN}

En este trabajo se analizan los cambios en el paisaje del viñedo (superficie, fragmentación y formas) entre 1956 y 2000 en La Rioja Alta (España). Se ha cartografiado el viñedo en 1956, 1977 y 2000 a partir de fotografía aérea. La cartografía ha sido tratada con diferentes sistemas de información geográfica y programas estadísticos. Los resultados demuestran la expansión del viñedo por espacios de otros cultivos y, en menor medida, por ambientes topográficos menos favorables. El paisaje del viñedo tendió a la desfragmentación, creando manchas de mayor tamaño y formas ligeramente más regulares.

Palabras clave: Viñedo, estructura del paisaje, cambios de paisaje rural, La Rioja, España.

\section{ABSTRACT}

Vineyard landscape changes were studied (surface, fragmentation and forms) between 1956 and 2000 in La Rioja Alta (Spain). The vineyards were mapped in 1956, 1977 and 2000 from aerial photography. The cartography was analysed with various GIS and statistical programs. Results showed the vineyard expansion for other cultivated areas, and, to a

Fecha de recepción: octubre 2013.

Fecha de aceptación: mayo 2015. 
lesser extent, for difficult topographic environments. The vineyard landscape tended to the defragmentation, creating larger patches with slightly more regular shapes.

Keywords: Vineyard, landscape structure, rural landscape changes, La Rioja, Spain

\section{INTRODUCCIÓN}

Los paisajes del viñedo han experimentado notables transformaciones en las últimas décadas en todos los países productores, aunque especialmente en los de la Unión Europea. Estas transformaciones se justifican por la mecanización del medio rural y la apertura de muchas de las regiones productoras de vino desde mercados locales, regionales o nacionales hacia mercados internacionales, con el consiguiente incremento de la competencia entre los vinos (Barco et al., 2005; Alonso Santos et al., 2003; Fernández Portela et al., 2013). Hay que tener en cuenta también que el consumo de vino disminuyó en el mundo en las dos últimas décadas del pasado siglo de 284,3 M/Hl/año, como media en el quinquenio 197680 , a $220 \mathrm{M} / \mathrm{Hl} / \mathrm{año}$ en 2000. Desde entonces el consumo muestra una tendencia alcista con cifras de $235 \mathrm{M} / \mathrm{Hl} /$ año en 2006 y de $245 \mathrm{M} / \mathrm{Hl} /$ año en 2012, según las estadísticas de la OIV (Organización Internacional de la Viña y el Vino). El incremento del consumo se centra en los vinos de calidad, generalmente con denominación de origen prestigiosa, mientras que los de menos calidad mantienen una tendencia regresiva.

La evolución del consumo y la fuerte competencia del mercado han llevado a cambios importantes en la extensión y localización del viñedo, sistemas de laboreo o viduño; cambios incentivados en gran medida por la Organización Común del Mercado (OCM) vitivinícola, a la que pertenecen la práctica totalidad de los países productores. En Europa, en concreto, las medidas restrictivas para las nuevas plantaciones y las incentivadoras para arrancar viñedos viejos se inician en 1987, tras la crisis de excedentes y bajos precios de los años precedentes y de las «guerras» productivas y comerciales entre Italia y Francia. Los avances en este sentido fueron muy importantes con la aprobación de la OCM del vino de 1999, dentro del paquete de reformas de la Agenda 2000.

La Reforma de la OCM de $1999^{1}$ perseguía la consecución de un equilibrio entre la oferta y la demanda del vino comunitario, incluyendo entre sus normas la prohibición de plantar nuevas vides. No obstante, se asignó una pequeña cantidad de derechos de plantación para zonas en expansión comercial. Se insistió en la necesidad de elaborar inventarios o registros vitícolas para ejercer un mayor control sobre la producción; se continuó con primas al arranque en regiones con excedentes estructurales persistentes; y se llevaron a cabo otras medidas de regulación como los programas de reconversión (sustitución de viñedos en vaso por el emparrado, como principal medida) y reestructuración varietal, que tuvieron importantes repercusiones paisajísticas.

La Reforma de la OCM de 1999 fue sustituida por el Reglamento (CE) N 479/2008 del Consejo, de 29 de abril, que establecía una nueva OCM (la conocida como OCM de 2008)

1 (Reglamento CE N ${ }^{\circ}$ 1493/1999 del Consejo de 17 de mayo de 1999, que fue en su mayor parte transpuesto al ordenamiento jurídico español con la Ley de la Vid y del Vino: ley 24/2003 de 10 de Julio de la Viña y el Vino) 
del sector vitivinícola. Entre las medidas con mayor impacto territorial se incluyen la continuación de las políticas de reestructuración y reconversión, la implantación del arranque subvencionado, la supresión de las medidas de apoyo al mercado para destinar esos fondos a la mejora de la calidad, y el impulso al desarrollo rural con el fin de facilitar la instalación de jóvenes agricultores. Además, las ayudas concedidas a la mecanización del cultivo explican el fuerte impulso de una nueva tipología de paisaje vitivinícola: el viñedo en espaldera (Cañizares Ruiz y Ruiz Pulpón, 2014).

Lógicamente, las OCM de 1999 y 2008 han introducido importantes cambios en los paisajes de viñedo. En España, por ejemplo, la superficie del viñedo ha descendido de 1.600.000 ha en 1986 a 967.055 ha en 2012, según el MAGRAMA. Por otro lado, se ha producido una migración desde los peores suelos, porque los mejores se destinaban a los cereales, a algunas de las mejores tierras, por el alto interés económico del viñedo para las explotaciones (Molinero et al., 2013).

Los rápidos cambios en los paisajes del viñedo español han impulsado su estudio, que además se ha visto incentivado por la aprobación del Convenio Europeo del Paisaje (Florencia, 2000), el reconocimiento de algunos de los paisajes del viñedo por la UNESCO (Cleere, 2004), la consideración del paisaje como un recurso socioeconómico ligado a su valor estético y la dinamización del enoturismo. Tales estudios analizan los cambios recientes (casi siempre posteriores al año 2000) del paisaje atendiendo a su papel socioeconómico y a su valor estético. Algunos valoran el papel de los cambios en la gestión y de las medidas reguladoras de las denominaciones de origen y las OCM de 1999 y 2008, destacando las reestructuraciones con el arranque de cepas, el cultivo en espaldera, la posibilidad del riego y las nuevas especies varietales. Sin ánimo de ser exhaustivos, es recomendable leer los trabajos de Castelló et al. (2010) sobre los viñedos de Aragón, de Molinero (2011) sobre los de Castilla y León, de Molleví (2014) sobre el Priorat, de Miramontes Carballeda (2014) sobre el paisaje vitícola en la Galicia mediterránea, de Mata Olmo y Ferrer Jiménez (2014) sobre los viñedos manchegos del Campo de San Juan, de Luginbühl y Villa Díaz (2014) sobre viñas y lagares en las campiñas béticas y de Cañizares Ruiz y Ruiz Pulpón (2014) sobre la evolución del paisaje del viñedo en Castilla-La Mancha. Resulta imprescindible, la lectura del trabajo de Molinero et al. (2013) sobre el conjunto de los viñedos españoles. Ruiz Pulpón (2010 y 2013) estudia las consecuencias en el paisaje de los programas de reconversión, centrándose en la expansión de los viñedos con posibilidades de riego y de los cultivados en espaldera.

El viñedo del Rioja y sus paisajes también han cambiado mucho desde los años ochenta del pasado siglo, mostrando en algunos casos la misma evolución que otros viñedos españoles (incremento del viñedo regado y del cultivo en espaldera), pero difiriendo en su evolución superficial y en la casi nula renovación varietal. Frente al retroceso de la mayor parte de los viñedos españoles (por ejemplo, en Castilla-La Mancha se pierden más de 130.000 ha desde al año 2000, como efecto de la retirada de viñas en secano y de las políticas de arranque subvencionado (Cañizares Ruiz y Ruiz Pulpón, 2014), el Rioja experimenta una considerable expansión, pasando de 38.349 ha en 1983 a 62.153 ha en 2012 (Consejo Regulador de la Denominación de Origen Calificada Rioja, en adelante DOCaRioja). Por otro lado, el enoturismo adquiere cierta importancia en los últimos 10-20 años, incorporando a la tradicional visita a las bodegas itinerarios por los paisajes del viñedo y 
el conocimiento del laboreo de la viña (Elías, 2009). Las publicaciones sobre el paisaje del Rioja proliferan en los últimos años, tratando aspectos sociales, antropológicos y turísticos (Elías, 2006 y 2014), patrimoniales (Palacios García, 2014), o los cambios en la fragmentación del paisaje, los marcos de plantación, el parcelario y los cambios en los sistemas de laboreo para adaptarse al mercado del vino y a las reestructuraciones promovidas desde las administraciones (Lasanta y Ruiz-Flaño, 2014).

Estos estudios se basan en estadísticas oficiales desde los años ochenta del siglo XX (las anteriores son menos fiables) y en la observación, constituyendo destacadas aportaciones al conocimiento de los paisajes del Rioja. Sin embargo, se plantean algunas cuestiones: i) las relaciones entre viñedos, suelos y topografía, de interés para la conservación del suelo y los laboreos (Arnáez et al., 2006) o ii) las causas que justifican la dinámica del Rioja. En este sentido, ¿los cambios responden sólo a la evolución reciente del mercado y a la legislación (OCM de 1999 y 2008)? o ¿se producían cambios en las décadas precedentes como respuesta a la mecanización y a los nuevos sistemas de laboreo? No cabe ninguna duda de que conocer el paisaje previo al actual tiene su interés, ya que todo paisaje mantiene huellas del pasado, con consecuencias ambientales, estéticas y en la biodiversidad. Los paisajes agrarios son resultado de la interacción entre el medio físico y el trabajo de generaciones de agricultores, con complementos no materiales en su gestación y desarrollo. Esta visión histórica del paisaje nos lleva a entenderlo como un concepto global en el que se suceden interacciones de todo tipo a lo largo del tiempo. Sólo una concepción holística puede explicar la complejidad de los paisajes.

Este trabajo se centra en una comarca de la DOCaRioja donde la vitivinicultura tiene una honda tradición que enraíza en los romanos, con distintas fases de decadencia y otras de expansión a lo largo de la Historia. Tras la crisis de la filoxera, a finales del siglo XIX, resurge con fuerza, adoptando el método bordolés de elaboración de vinos para abastecer al mercado francés (Huetz de Lemps, 1967). Desde mediados del siglo XX la expansión superficial ha sido sostenida, paralela a la mayor presencia del Rioja en los mercados internacionales (Barco, 2008).

El objetivo principal de este trabajo es estudiar la evolución, desfragmentación y forma de las manchas del viñedo, variables destacadas de la estructura del paisaje, en un lapso temporal de 44 años (desde 1956 a 2000). Es un paso previo para, en el futuro, analizar los cambios desde el año 2000 a la actualidad y, de este modo, valorar mejor las transformaciones introducidas por la reciente dinámica del mercado y por la legislación. Por otro lado, con el estudio se pretende aportar información sobre las interrelaciones entre medio físico y paisaje.

\section{II. ÁREA DE ESTUDIO}

El estudio se ha llevado a cabo en La Rioja Alta (España), en las comarcas de Haro y Nájera, situadas en el sector más occidental de la Depresión del Ebro (Fig. 1). La Sierra de Obarenes-Cantabria delimita con dirección NW-SE su borde septentrional y la vertiente norte del Sistema Ibérico el borde meridional. La altitud media del área de estudio oscila entre 500 y 600 m.s.n.m, alcanzándose la cota máxima en la Sierra del Toloño (1271 m.s.n.m). Los materiales de la zona corresponden a litologías detríticas del Terciario, orde- 
nadas en las facies Haro (areniscas amarillas, lutitas y arcillas grises) y Nájera (areniscas rojas y arcillas margosas), y del Cuaternario (glacis y terrazas). Los suelos predominantes son calcisoles de textura franca y arenosa (Gómez Miguel, 2006), con una topografía de suaves pendientes y formas ligeramente alomadas o llanas. El clima es de tipo mediterráneo con influencias atlánticas, con una precipitación anual de 400-500 mm y valores máximos en primavera e invierno. La temperatura media anual oscila entre los $12-13^{\circ} \mathrm{C}$, registrándose una moderada amplitud térmica: $4-6^{\circ} \mathrm{C}$ en diciembre-enero y $20-21^{\circ} \mathrm{C}$ en julio-agosto.

En el año 2010, el viñedo, con 21.247 hectáreas, era el cultivo principal seguido por el cereal, con 10.924 hectáreas. El viñedo constituye la matriz del paisaje y el resto está completado por parcelas de cereal, patatas, guisantes y pequeños eriales en las zonas de contacto entre distintos niveles de acumulación. El 80,2\% del viñedo (17.032 ha) se localiza en tierras de secano y el resto en regadío (4.215 ha); se trata en el último caso de parcelas con riego localizado, que han sido plantadas desde el año 2003 o viñedos más antiguos a los que se ha llevado agua para el riego. El tamaño de las parcelas es pequeño, dominando las de menos de 1 ha, si bien la tendencia es al incremento del tamaño de las parcelas, con frecuencia ligado a la compra o arrendamiento de viñedos por las grandes bodegas y a la normativa para acceder a las subvenciones de reestructuración.

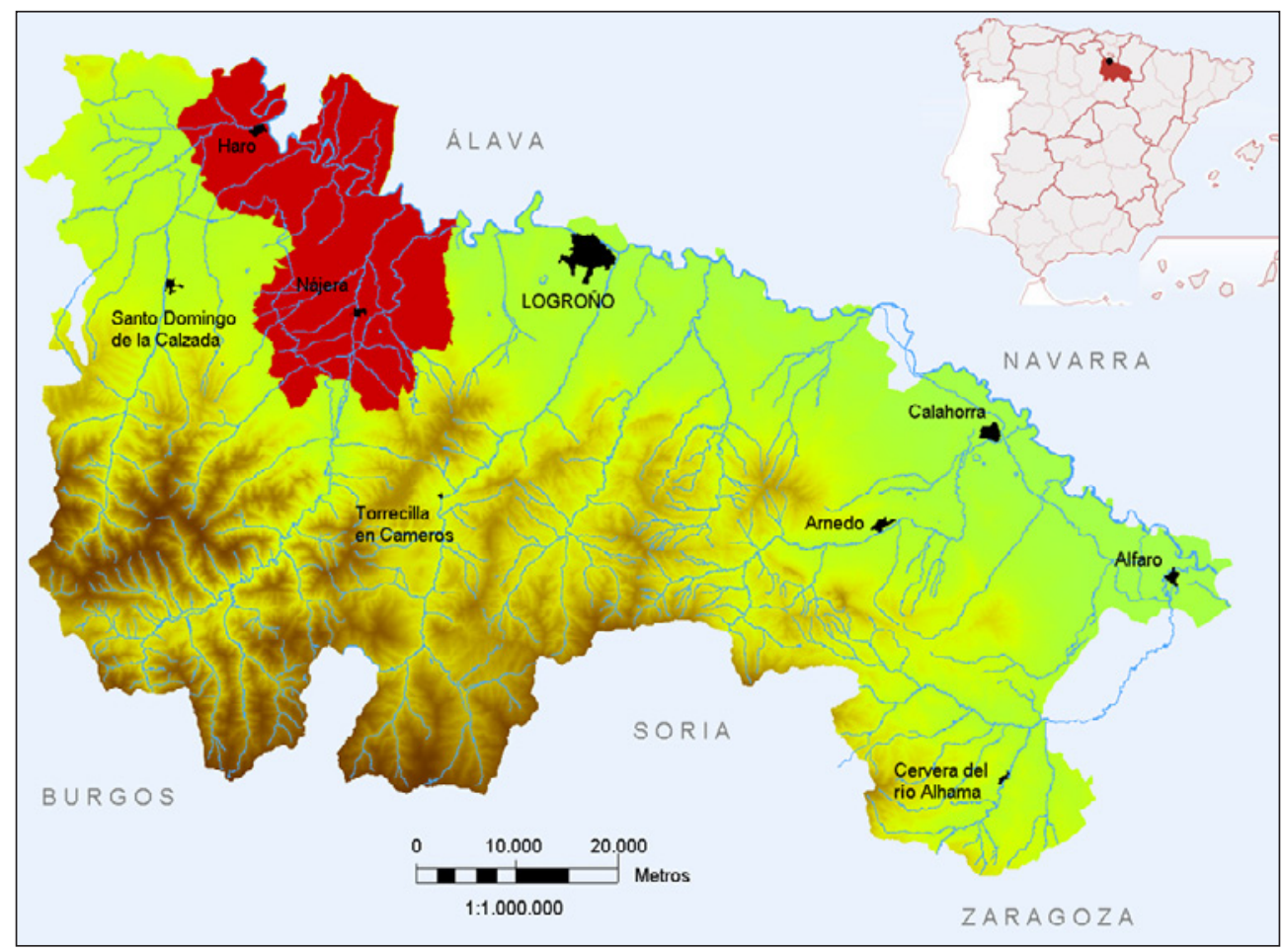


Los viñedos se localizan preferentemente entre 400 y 600 m.s.n.m., intervalo en el que se concentra el $73 \%$ de todo el viñedo. Suele asentarse sobre superficies casi planas o ligeramente inclinadas de glacis y terrazas antiguas, aunque es frecuente la presencia de viñas en laderas relativamente pendientes entre distintos niveles de glacis. Por encima del $20 \%$ de pendiente se cultiva el 18,7\% del viñedo de La Rioja Alta (Arnaéz et al., 2006). Es un paisaje de viñas jóvenes con menos del 15\% de vides que superen los 40 años, edad a partir de la cual se considera que la producción se reduce a la mitad. El rejuvenecimiento de las plantaciones tuvo lugar preferentemente entre finales de los años ochenta y 2000, coincidiendo con la buena coyuntura económica que vivió en ese periodo el Rioja (en 1988 el Kg de uva negra se pagó al agricultor a 0,60 Euros, precio que ascendió hasta alcanzar la máxima cotización en 1999 con 1,95 Euros). Desde el año 2000 el precio ha oscilado entre 0,7 y 0,96 Euros/Kg, lo que unido a la legislación ha ralentizado el ritmo de plantación desde el año 2000, de forma que en 2011 en la DOCaRioja sólo 487 ha (el 0,76\%) cuentan con vides de menos de 10 años.

En algunos municipios del área de estudio el viñedo es casi un monocultivo, ocupando al menos el $60 \%$ del territorio (San Asensio y Cenicero, por ejemplo). Su alta extensión se explica por las buenas condiciones ambientales para producir vinos de calidad, pero también porque a raíz de la crisis de la filoxera la comarca se vio favorecida por su proximidad y buena comunicación con importantes mercados españoles (País Vasco, Madrid) y europeos (Francia, fundamentalmente), lo que favoreció la instalación de grandes bodegas desde finales del siglo XIX (Huetz de Lemps, 1967).

\section{MATERIALES Y MÉTODOS}

Ya se ha indicado que el objetivo principal del trabajo es estudiar la evolución, desfragmentación y forma de las manchas del viñedo desde 1956 a 2000. El año 2000 es una fecha clave para identificar los cambios previos a la OCM de 1999. Hay que recordar que, después del año 2000, los programas de reconversión y reestructuración de las OCM de 1999 y 2008 tienen gran importancia en el paisaje: modificaciones en los sistemas de cultivo y puesta en regadío de algunos viñedos (Ruiz Pulpón, 2010 y 2013). Es por ello que se haya optado por finalizar el análisis de este trabajo en el año 2000.

El trabajo se ha apoyado en el estudio de la superficie del viñedo en tres fechas: 1956, 1977 y 2000. Para ello se dispuso de fotografías áreas en blanco y negro a escala 1:33000 (año 1956) y a escala 1:18000 (años 1977 y 2000). Se escanearon los fotogramas con la máxima resolución, se georreferenciaron y se procedió a cartografiar con detalle las parcelas de viñedos con la ayuda de un software SIG (ArcView). Las imágenes utilizadas cubrieron un total de $517,37 \mathrm{~km}^{2}$. Una vez generados los mapas, los datos fueron exportados a un software SIG raster (MFWorks 3.0) y a Fragstats 3.3 (McGarigal y Marks, 1995; McGarigal et al., 2002) con el objeto de poder analizar y visualizar los datos espacialmente.

El SIG raster permite visualizar la distribución del viñedo en distintas fechas y generar un mapa de síntesis. Con el objeto de homogenizar y aligerar la base de datos, la cartografía original de $5 \mathrm{~m} /$ pixel fue transformada a una resolución de $20 \mathrm{~m} /$ pixel.

Fragstats es un programa de análisis geoestadístico especializado en la fragmentación y métrica de paisajes. Calcula una serie de índices que muestran las propiedades del paisaje: tamaños de las áreas, configuración y dispersión, fragmentación, etc. Para conocer las for- 
mas de las unidades del viñedo se empleó el Landscape Shape Index (LSI) (McGarigal et al., 2002). Cuando LSI es igual a 1, el paisaje estaría formado por una única tesela o unidad circular (formato vectorial) o cuadrada (formato raster). A medida que LSI supera el valor 1 el paisaje se vuelve más irregular y complejo en su forma.

Para completar la información y relacionar la superficie de viñedo con variables geomorfológicas y topográficas se elaboró un mapa geomorfológico que incluyó formas de sedimentación: terrazas altas (niveles 6 a 9), terrazas medias (niveles 4 a 5), terrazas bajas (niveles 2 a 3), terraza 1 y lechos de inundación, glacis altos (niveles 6 a 12), glacis medios (niveles 4 a 5), glacis bajos (niveles 2 a 3), glacis 1 y conos aluviales; formas fluviales: paleocanales y valles en cunas; formas de vertiente: vertientes regularizadas; y áreas de erosión: cárcavas, rigolas y badlands. También se dispuso de un mapa digital del terreno (MDT), con una resolución de $5 \mathrm{~m} /$ pixel, a partir de cual se obtuvo una cartografía de altitudes, pendientes y exposiciones.

Los archivos cartográficos fueron exportados al programa de análisis estadístico SPSS 15 para, de este modo, poder trabajar con bases de datos de gran tamaño.

\section{RESULTADOS}

\section{IV.1. Evolución de la superficie del viñedo}

El periodo 1956-1977 supone el paso de una agricultura tradicional, basada en el policultivo, a otra más intensiva, mecanizada y organizada en torno a unos cultivos muy orientados al mercado. Por lo que respecta al caso concreto de La Rioja, en el periodo comprendido entre finales de los años sesenta y mediados de los setenta se produce una crisis agrícola en la que la normalización de la industria vinícola, la escasa rentabilidad del viñedo y las deficientes cosechas favorecieron el abandono de los viñedos menos productivos y más marginales. En las tablas 1,2, 3 y 4 puede comprobarse cómo en 1977 se dejó de cultivar el viñedo localizado en áreas con problemas de erosión (un $28 \%$ con respecto a 1956), por encima de los $700 \mathrm{~m}$ de altitud (un $48,2 \%$ en la franja de 700 a $800 \mathrm{~m}$ y un $100 \%$ en la franja de 800 a 900 m), en pendientes superiores al $40 \%$ (un $9,1 \%$ entre un $40-80 \%$ de gradiente) y en orientaciones septentrionales ( $\mathrm{N}$ y NE). Por otro lado, el viñedo es sustituido en áreas favorables por otros cultivos con buenas salidas a los mercados y fácil mecanización (cereales y tubérculos). Así, las mismas tablas muestran la desaparición de viñedos en terrazas medias y bajas (un 25,8 y $27,1 \%$, respectivamente), y laderas con pendientes inferiores al $5 \%$ (un 15,7\%). En definitiva, en 1956 se contabilizaban 12.802 ha de viñedo en el área de estudio que quedaron reducidas a 11.490 ha en 1977 .

Las décadas de los 80 y 90 suponen una rápida recuperación de la superficie del viñedo. La imposición de una economía de mercado en el mundo rural, el aumento de la demanda exterior, la modernización de las estructuras productivas, los elevados precios de la uva y los sucesivos años de buenas cosechas impulsan el incremento del viñedo, de modo que en el año 2000 su superficie ya alcanzaba en el área de estudio 15.497 ha (un incremento del 34,8\% con respecto a 1977). La superficie aumentó de forma bastante homogénea en el conjunto del territorio, sin embargo los incrementos han sido más notorios en las terrazas y glacis altos $(58,3 \%$, en las primeras, y $41,2 \%$ en los segundos), así como en los valles en cuna $(61,8 \%)$ 
y vertientes regularizadas, espacios dedicados tradicionalmente al cereal (Tabla 1). También en esta expansión ha sido necesario volver a recuperar superficies situadas por encima de los $700 \mathrm{~m}$ pero especialmente por debajo de los $500 \mathrm{~m}$ (42\%) (Tabla 2). En las tablas 3 y 4 se observa cómo los incrementos de superficie del viñedo se han conseguido en áreas con una pendiente relativamente elevada y en laderas solanas. Es decir, el espacio incorporado al viñedo o bien ha sido obtenido a costa de otros cultivos o ha ocupado territorios de condiciones topográficas difíciles, pero rentables dados los rendimientos y beneficios de este cultivo.

Tabla 1

DISTRIBUCIÓN Y EVOLUCIÓN DE LA SUPERFICIE DEL VIÑEDO SEGÚN LAS PRINCIPALES FORMAS DEL RELIEVE PARA LOS PERIODOS 1956, 1977 Y 2000

\begin{tabular}{|l|c|c|c|c|c|}
\hline \multicolumn{1}{|c|}{ Formas del relieve } & 1956 (ha) & 1977 (ha) & $\begin{array}{c}\text { Evolución } \\
1956-77 \\
(\%)\end{array}$ & 2000 (ha) & $\begin{array}{c}\text { Evolución } \\
1977-00 \\
(\%)\end{array}$ \\
\hline Terrazas altas (6-9) & 822 & 746,7 & $-9,2$ & $1.182,3$ & $+58,3$ \\
\hline Terrazas medias (4-5) & 536,2 & 398 & $-25,8$ & 617,4 & $+55,1$ \\
\hline Terrazas bajas (3-2) & 1.822 & 1.329 & $-27,1$ & $1.754,9$ & $+32,0$ \\
\hline Terraza 1 y lecho inundación & $1.371,6$ & 1.276 & $-7,0$ & $1.764,2$ & $+38,3$ \\
\hline Glacis altos (12-6) & 207,7 & 205,3 & $-1,2$ & 289,9 & $+41,2$ \\
\hline Glacis medios (5-4) & $2.454,3$ & $2.459,8$ & $+0,2$ & $3.099,3$ & $+26,0$ \\
\hline Glacis bajos (3-2) & $2.352,2$ & $2.074,8$ & $-11,8$ & $2.545,5$ & $+22,7$ \\
\hline Glacis 1 y conos & 628,3 & 542 & $-13,7$ & 701,8 & $+29,5$ \\
\hline Paleocanales y valles en cuna & 50,4 & 45,8 & $-9,1$ & 74,1 & $+61,8$ \\
\hline Vertientes regularizadas & 24,4 & 22,4 & $-8,2$ & 52,5 & $+134,4$ \\
\hline Rigolas y cárcavas & 2,5 & 1,8 & $-28,0$ & 2,8 & $+55,6$ \\
\hline Otros (areniscas terciarias) & $2.530,4$ & $2.388,4$ & -5.6 & $3.412,3$ & $+42,8$ \\
\hline Total & 12.802 & 11.490 & & 15.497 & \\
\hline
\end{tabular}

Fuente: elaboración propia.

Tabla 2

DISTRIBUCIÓN Y EVOLUCIÓN DE LA SUPERFICIE DEL VIÑEDO SEGÚN LAALTITUD PARA LOS PERIODOS 1956, 1977 Y 2000

\begin{tabular}{|l|c|c|c|c|c|}
\hline Altitud (m) & $1956(\mathrm{ha})$ & $1977(\mathrm{ha})$ & $\begin{array}{c}\text { Evolución } \\
1956-77 \\
(\%)\end{array}$ & 2000 (ha) & $\begin{array}{c}\text { Evolución } \\
1977-00 \\
(\%)\end{array}$ \\
\hline $400-500$ & $4.442,2$ & 4.014 & $-9,6$ & $5.699,7$ & $+42,0$ \\
\hline $500-600$ & $6.430,3$ & 5.727 & $-10,9$ & $7.403,2$ & $+29,3$ \\
\hline $600-700$ & $1.873,4$ & 1.721 & $-8,1$ & $2.332,8$ & $+35,5$ \\
\hline $700-800$ & 54 & 28 & $-48,2$ & 61,3 & $+118,9$ \\
\hline $800-900$ & 2,1 & & $-100,0$ & & \\
\hline Total & 12.802 & 11.490 & & 15.497 & \\
\hline
\end{tabular}

Fuente: elaboración propia. 
Tabla 3

DISTRIBUCIÓN Y EVOLUCIÓN DE LA SUPERFICIE DEL VIÑEDO SEGÚN LA PENDIENTE PARA LOS PERIODOS 1956, 1977 Y 2000

\begin{tabular}{|l|c|c|c|c|c|}
\hline Pendiente (\%) & 1956 (ha) & $1977(\mathrm{ha})$ & $\begin{array}{c}\text { Evolución } \\
1956-77(\%)\end{array}$ & $2000(\mathrm{ha})$ & $\begin{array}{c}\text { Evolución } \\
1977-00(\%)\end{array}$ \\
\hline $0-5$ & 6.330 & $5.338,5$ & $-15,7$ & $5.824,7$ & $+9,1$ \\
\hline $5-10$ & $3.352,7$ & $3.091,3$ & $-7,8$ & 4.809 & $+55,5$ \\
\hline $10-20$ & $2.467,5$ & $2.384,1$ & $-3,4$ & $3.752,1$ & $+57,4$ \\
\hline $20-40$ & 633 & 659 & $+4,1$ & $1.073,1$ & $+62,8$ \\
\hline $40-80$ & 18,7 & 17 & $-9,1$ & 38 & $+123,5$ \\
\hline$>80$ & 0,1 & 0,07 & -30 & 0,05 & $-28,6$ \\
\hline Total & 12.802 & 11.490 & & 15.497 & \\
\hline
\end{tabular}

Fuente: elaboración propia.

Tabla 4

DISTRIBUCIÓN Y EVOLUCIÓN DE LA SUPERFICIE DEL VIÑEDO SEGÚN LA EXPOSICIÓN PARA LOS PERIODOS 1956, 1977 Y 2000

\begin{tabular}{|l|c|c|c|c|c|}
\hline Exposición & 1956 (ha) & $1977(\mathrm{ha})$ & $\begin{array}{c}\text { Evolución } \\
1956-77(\%)\end{array}$ & $2000(\mathrm{ha})$ & $\begin{array}{c}\text { Evolución } \\
1977-00(\%)\end{array}$ \\
\hline $\mathrm{N}$ & $2.259,4$ & $1.938,7$ & $-14,2$ & $2.462,1$ & $+27,0$ \\
\hline $\mathrm{NE}$ & $1.817,5$ & $1.519,5$ & $-16,4$ & $1.983,2$ & $+30,5$ \\
\hline $\mathrm{E}$ & $1.576,0$ & $1.396,5$ & $-11,4$ & $1.835,6$ & $+31,4$ \\
\hline $\mathrm{SE}$ & $1.521,0$ & $1.426,5$ & $-6,2$ & $1.933,4$ & $+35,5$ \\
\hline $\mathrm{S}$ & $1.139,2$ & $1.037,7$ & $-8,9$ & $1.512,5$ & $+45,7$ \\
\hline $\mathrm{SW}$ & 913,5 & 879 & $-3,8$ & $1.384,5$ & $+57,5$ \\
\hline $\mathrm{W}$ & 1.309 & $1.234,7$ & $-5,6$ & 1.734 & $+40,4$ \\
\hline NW & $2.222,6$ & 2.021 & $-9,1$ & 2.601 & $+28,7$ \\
\hline Horizontal & 43,7 & 36,4 & $-16,7$ & 50,5 & $+38,7$ \\
\hline Total & 12.802 & 11.490 & & 15.497 & \\
\hline
\end{tabular}

Fuente: elaboración propia.

\section{IV.2. Estructura del espacio del viñedo}

Los descensos o incrementos de superficie cultivada implicaron modificaciones en el paisaje del viñedo. En la Tabla 5 se aprecia que la relación entre la creciente superficie del viñedo es inversa al número de áreas o unidades cultivadas (fragmentos, manchas, parches o teselas) y su tamaño medio. En 1956 se disponía en la zona de estudio de 5.125 áreas homogéneas de cultivo del viñedo (2,49 ha/unidad). En el año 2000 la extensión del viñedo es mucho mayor (15.497 ha), pero el número de unidades disminuye a $3.348 \mathrm{y}$ aumenta su tamaño (4,62 ha). En síntesis, la tendencia general se caracteriza por la progresiva concentración de la superficie del viñedo en un menor número de manchas, de tal modo que las teselas van adquiriendo mayores dimensiones. 
Tabla 5

CARACTERÍSTICAS DE LAS ÁREAS O UNIDADES CULTIVADAS DE VIÑEDO EN EL ÁREA DE ESTUDIO

\begin{tabular}{|c|c|c|c|c|c|}
\hline Año & $\begin{array}{c}\text { Superficie de } \\
\text { viñedo (ha) }\end{array}$ & $\begin{array}{c}\text { \% del área de } \\
\text { estudio }\end{array}$ & $\mathrm{N}^{\text {o de unidades }}$ & $\begin{array}{c}\text { Densidad de } \\
\text { las unidades } \\
\left(\mathrm{km}^{2}\right)\end{array}$ & $\begin{array}{c}\text { Superficie } \\
\text { media de las } \\
\text { unidades (ha) }\end{array}$ \\
\hline 1956 & 12.802 & 24,7 & 5.125 & 0,10 & 2,49 \\
\hline 1977 & 11.490 & 22,2 & 4.696 & 0,11 & 2,44 \\
\hline 2000 & 15.497 & 29,9 & 3.348 & 0,15 & 4,62 \\
\hline
\end{tabular}

Fuente: elaboración propia.

La tendencia evolutiva del paisaje de la vid, caracterizada por la disminución de la dispersión espacial, se puede observar en las Fig. 2 y 3 . Se trata de cartografías del viñedo en las diferentes fechas de estudio y con distinto tamaño de pixel. Los mapas de $20 \mathrm{~m} /$ pixel de resolución permiten intuir la tendencia a la concentración con el paso del tiempo. De hecho, en el mapa correspondiente a 2000 las áreas más vitícolas del área de estudio -las más próximas al eje del Ebro- presentan una mayor densificación. Para ayudar a interpretar visualmente el comportamiento espacial de la superficie del viñedo, la cartografía mencionada ha sido transformada a un tamaño de $500 \mathrm{~m} /$ pixel. Los resultados evidencian que los mapas correspondientes a los años 1956 y 1977 presentan un territorio vitícola más disperso y heterogéneo. En el mapa del año 2000, la homogeneización y concentración del paisaje de la vid son más evidentes con una cierta tendencia al monocultivo. Al mismo tiempo, se observa que en la última fecha desaparecen muchas manchas de reducido tamaño presentes en 1956.
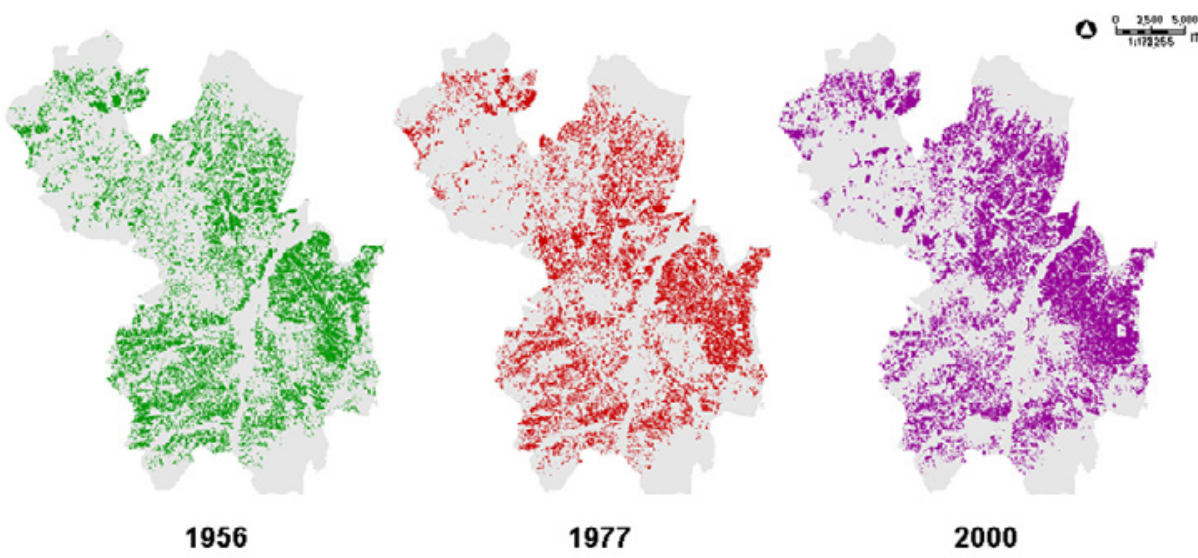

Las transformaciones de la estructura del paisaje agrario de la vid en los 44 años de estudio han resultado ser más complejas de lo que se deduce a través de las cartografías (Tabla 6). Las $5.233,3$ ha de viñedos que han permanecido inalteradas en las tres fechas analizadas 
configuran fragmentos de 0,84 ha mientras que, por el contrario, la superficie de viñedo abandonada, tanto en el periodo 1956-77 como en el 1977-00, se identificaría con teselas de menores dimensiones: 0,39 y 0,28 ha. Las manchas de viñedos incorporadas con posterioridad a 1956 o 1977 han configurado manchas de mayores dimensiones: 0,47 y 0,56 ha. Así, pues, con el paso del tiempo, el paisaje del viñedo se ha ido densificando, haciéndose monocultivo en determinados municipios donde se ha incrementado su superficie y se han ido configurando áreas de viñedo de mayores dimensiones.

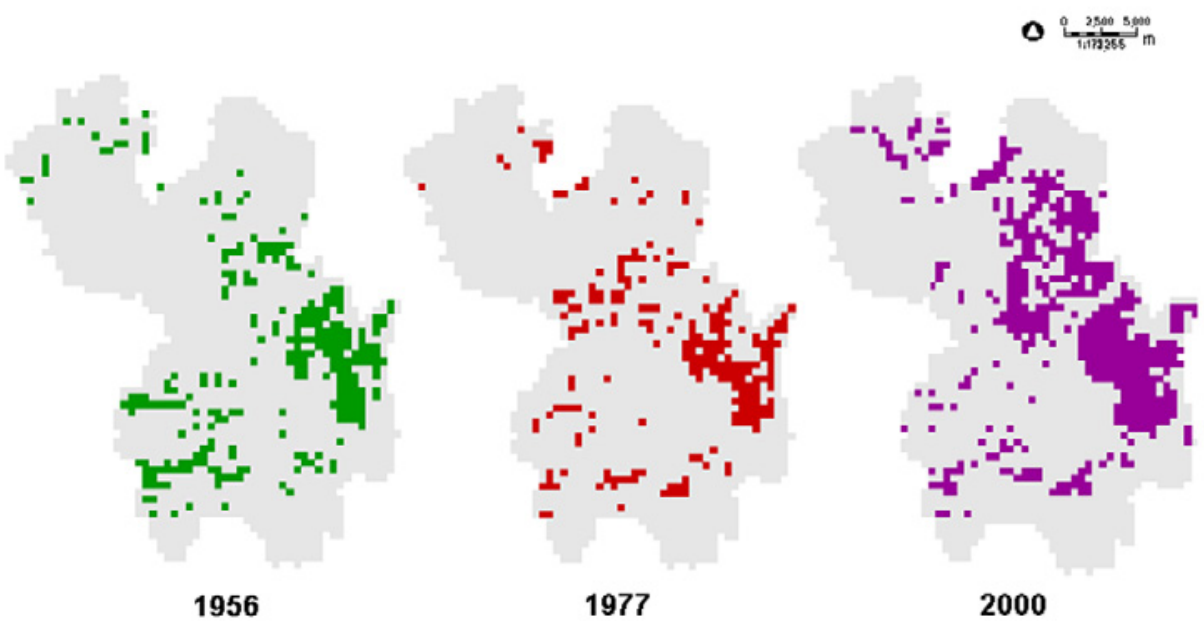

Tabla 6

CARACTERÍSTICAS DE LAS ÁREAS O UNIDADES CULTIVADAS DE VIÑEDO SEGÚN SU COMPORTAMIENTO TEMPORAL

\begin{tabular}{|l|c|c|c|c|c|}
\hline & $\begin{array}{c}\text { Superficie } \\
\text { (ha) }\end{array}$ & $\begin{array}{c}\mathrm{N}^{\text {o de }} \\
\text { áreas }\end{array}$ & $\begin{array}{c}\text { Densidad } \\
\text { de las unidades } \\
\left(\mathrm{km}^{2}\right)\end{array}$ & $\begin{array}{c}\text { Superficie } \\
\text { media de } \\
\text { las unidades } \\
\text { (ha) }\end{array}$ & $\begin{array}{c}\text { Superficie } \\
\text { máxima de las } \\
\text { unidades (ha) }\end{array}$ \\
\hline Sin cambios & $5.233,3$ & 6.166 & 0,083 & 0,84 & 122,16 \\
\hline Abandonadas desde 1956 & $3.171,2$ & 8.118 & 0,063 & 0,39 & 29,8 \\
\hline Abandonadas desde 1977 & $1.660,7$ & 5.854 & 0,088 & 0,28 & 9,84 \\
\hline Incorporadas desde 1956 & $2.889,1$ & 6.099 & 0,084 & 0,47 & 50,2 \\
\hline Incorporadas desde 1977 & $4.948,3$ & 8.746 & 0,059 & 0,56 & 86,88 \\
\hline
\end{tabular}

Fuente: elaboración propia. 


\section{IV.3. Morfometría de las unidades del viñedo}

Uno de los criterios aplicados en el reconocimiento del paisaje es la diferenciación entre las formas simples y bien definidas, de origen antrópico, de aquellas otras formas más difusas e irregulares propias de los paisajes naturales (Burel y Baudry, 2002). La estructura del mosaico paisajístico de La Rioja Alta tiene un claro origen humano, basado en parcelaciones de pequeñas dimensiones y formas rectangulares o poliédricas. En menor medida, se constata la existencia de parcelas irregulares y complejas.

Tabla 7

ÍNDICE LSI (LANDSCAPE SHAPE INDEX)

\begin{tabular}{|c|c|c|c|}
\hline Año & Media & Mediana & Desviación estándar \\
\hline 1956 & 1,56 & 1,33 & 0,99 \\
\hline 1977 & 1,55 & 1,28 & 0,99 \\
\hline 2000 & 1,57 & 1,28 & 1,1 \\
\hline
\end{tabular}

Fuente: elaboración propia.

En la Tabla 7 se muestran los resultados del índice morfométrico LSI en las tres fechas estudiadas. La mediana más baja del índice se corresponde con la estructura del paisaje de los años 1977 y 2000, con un valor de 1,28. Por el contrario, la morfometría del año 1956 revela un valor levemente superior $(1,33)$. La desviación estándar presenta en el año 1956 un valor de 0,99 mientras que en 2000 era de 1,1. Estos datos ponen de manifiesto que las unidades del viñedo no han cambiando sustancialmente en cuanto a su forma en el periodo estudiado, aunque evolucionan lentamente hacia una geometría más simple y proporcionada. A esta conclusión también se llega a partir de la medición de los perímetros de las unidades de viñedo (Tabla 8). En el año 2000 la suma de los perímetros de las áreas es inferior a la de las fechas anteriores. La densidad perímetro/territorio es claramente desfavorable en el paisaje del año 1956.

En cualquier caso, se reitera que, aunque la estructura del paisaje del viñedo en 1956 presentaba mayor fragmentación, LSI no sugiere un cambio contrastado y claro en las formas de las unidades del viñedo. De hecho, analizando la cartografía se aprecian tanto en 1956 como en 2000 numerosas parcelas individualizadas, generalmente muy alargadas y de pequeño tamaño, claro ejemplo de un modelo minifundista donde predominan las pequeñas y medianas explotaciones de propiedad familiar.

Tabla 8

PERÍMETROS DE LAS ÁREAS DE VIÑEDO

\begin{tabular}{|c|c|c|}
\hline Año & Perímetro de las áreas $(\mathrm{m})$ & Densidad $\left(\mathrm{m}^{2}\right)$ \\
\hline 1956 & 5.542 .580 & 0,0107 \\
\hline 1977 & 5.028 .300 & 0,0097 \\
\hline 2000 & 4.924 .760 & 0,0095 \\
\hline
\end{tabular}

Fuente: elaboración propia. 


\section{DISCUSIÓN Y CONCLUSIONES}

La superficie del viñedo en La Rioja Alta se ha incrementado en el periodo estudiado, pasando de representar el $24,7 \%$ de la superficie de la comarca en 1956 al 29,9\% en el año 2000. En este proceso, no obstante, hay que distinguir un primer periodo, que abarca desde 1956 a 1977, en el que se producen abandonos de la superficie cultivada, y otro, 1977-2000, donde, por el contrario, se recuperan tierras de viñedos.

La cartografía revela que el viñedo tiende a concentrarse en determinados sectores. De hecho, es en las áreas más próximas al río Ebro y al curso bajo del Najerilla donde se aprecian las mayores extensiones de vid. La expansión de la superficie vitícola del periodo 19772000 ha creado una mayor homogeneidad del paisaje del viñedo, acercándose al monocultivo en algunos municipios. A la vez que se ha ido configurando manchas de mayores dimensiones, habiéndose eliminado en las últimas décadas muchas de las parcelas aisladas o diminutas. Sin embargo, no parece haber variado sustancialmente las formas de estas unidades.

En definitiva, en 2000 tenemos un paisaje del viñedo menos fragmentado que en las décadas anteriores, un proceso que también ha tenido lugar en otros paisajes europeos (Jongman, 2002). Ello resulta lógico si tenemos en cuenta el creciente protagonismo de la vitivinicultura en La Rioja. A partir de la información incluida en las estadísticas del Gobierno de la Rioja (Estadística Agraria Regional, 1987, 2001 y 2011), la aportación de la vitivinicultura a la Producción Final Agraria se ha ido incrementado, siendo del 13,6\% en 1986 y del 29,4\% en 2001. En 2010 ya era del 34,3\%. Su mayor peso económico se explica -entre otras razones- por el avance superficial del viñedo frente a otros cultivos, lo que disminuye el mosaico de usos agrícolas. El paisaje tiende a perder diversidad por la disminución del número de cultivos. Es una consecuencia de la ampliación de los mercados y de la necesidad de pasar a economías de escala en las que es necesario competir nacional e internacionalmente. La evolución de los sistemas de laboreo con el incremento de la mecanización también ha contribuido a eliminar bordes de parcelas para fusionarlas y racionalizar los laboreos. Por último, Lasanta y Ruiz-Flaño (2014) señalan que la unión de parcelas, derribando bancales y allanando ribazos, tiene mucho que ver con la jubilación de muchos agricultores y la compra de derechos de plantación por las grandes bodegas, lo que ha favorecido la concentración de los viñedos en menos explotaciones.

Es pronto aún para valorar cuáles son las principales consecuencias ambientales de la nueva estructura del paisaje, caracterizada por la desfragmentación. No obstante, la simplificación de paisajes complejos en ambientes de topografía ondulada o ligeramente accidentada suele ser un factor importante de erosión, sobre todo cuando esa simplificación implica además la disminución del número de parcelas debido a la concentración. En ese caso, se reducen los límites entre campos, que frenan localmente la erosión y producen zonas intermedias de sedimentación, limitando la exportación de materiales y la conectividad entre la parte alta y la baja de las laderas. Ruiz-Flaño et al. (2006) demostraron la importancia de los linderos y de los saltos entre parcelas, que interrumpen la erosión laminar y la continuidad de las incisiones, favoreciendo la existencia de un mosaico de ambientes geomorfológicos poco continuos. Raclot et al. (2009), al estudiar los efectos de la escala en la erosión en viñedos del sureste de Francia, concluyeron que las tasas de erosión observadas a escala de cuenca fueron significativamente inferiores a las observadas a escala de parcelas individuales. Esto 
significa, en primer lugar, que la erosión en una cuenca no puede estimarse por la suma de la erosión en las parcelas, y, en segundo lugar, que dentro de una cuenca hay muchos sumideros o almacenes temporales de sedimento que interrumpen la accesibilidad de los materiales desde las parcelas a los cauces. En eso consiste la complejidad, es decir, la presencia de interruptores que dificultan la conexión hacia los cauces (Quiñonero-Rubio et al., 2013). La concentración de parcelas y la ampliación de las unidades de paisaje tienden a eliminar esos almacenes temporales. Una gestión adecuada del territorio debe tener en cuenta este problema y mantener un mínimo de diversidad que frene la conectividad entre laderas y cauces, una decisión imprescindible en cultivos propensos a la erosión del suelo cuando se instalan en laderas pendientes.

En relación con la erosión del suelo en viñedos hay que recordar también que Constantini (1992) ha llegado a la conclusión de que la calidad del vino y su productividad están en relación inversa a la erosión del suelo. Este problema se ha detectado incluso en algunas áreas centroeuropeas (Richter y Negendank, 1977). La apertura a grandes mercados internacionales ha supuesto también la reestructuración general del paisaje y de los sistemas de cultivo en algunas regiones vitivinícolas (Arnáez et al., 2012), con la incorporación de nuevas técnicas de nivelado de tierras a gran escala y la construcción de grandes terrazas para aprovechar laderas muy pendientes, lo que ha provocado destacados cambios en el paisaje y no pocos problemas ambientales (Cots-Folch et al., 2006; Ramos et al., 2007; Martínez-Casasnovas et al., 2010; Ramos y Martínez-Casasnovas, 2010).

El tema de la fragmentación del paisaje se ha estudiado sobre todo desde una perspectiva ecológica, por el destacado papel del tamaño de las manchas, de sus formas y de sus bordes en los índices de heterogeneidad de un territorio (Burel y Baudry, 2002). En espacios agrícolas, de dilatada ocupación humana, como es el caso del área de estudio, se considera que la disminución de la fragmentación favorece la desaparición de especies adaptadas a paisajes en mosaico, donde alternan diferentes cultivos y pequeñas áreas de eriales, que son sustituidas por otras más comunes que se ven favorecidas por manchas más extensas (Freemark y Merriam, 1986). Por otro lado, la eliminación de algunos bordes de campos suele implicar la desaparición de áreas que actúan como corredores ecológicos o green veining con implicaciones muy negativas para la biodiversidad (Grashof-Bokdam y Van Langevelde, 2004).

Desde hace 10-20 años, la vitivinicultura del Rioja ha añadido el enoturismo como nuevo producto. Entre otras actividades a realizar por los visitantes se incluye las visitas y excursiones por los viñedos, experimentando su poder de atracción una evolución creciente. El valor paisajístico, estético y cultural de los viñedos ha llevado a que el Gobierno de La Rioja considere el viñedo como Paisaje de Interés Cultural. Sin embargo, surgen algunas sombras tras los cambios del paisaje de las últimas décadas, ya que la tendencia creciente hacia la homogeneización, desfragmentación, expansión de plantaciones en espaldera, migración de viñedos en ladera hacia espacios llanos y más fértiles amenaza con transformar un paisaje singular, cultural y bello en un paisaje muy generalista, similar al de otros paisajes europeos de viñedos. Las condiciones ambientales y el laboreo de generaciones de agricultores originaron un paisaje diverso, rico en biodiversidad y estética, único y singular; un paisaje que ha evolucionado hacia la homogeneización impuesta por la mecanización y la legislación uniforme de la Unión Europea, lo que constituye una amenaza para el mantenimiento del paisaje cultural del viñedo. 


\section{REFERENCIAS}

ALONSO SANTOS, J.L., APARICIO AMADOR, L.J. y SÁNCHEZ HERNÁNDEZ, J.L. (2003): Los espacios vitivinícolas en Castilla y León: la evolución hacia un sistema productivo de calidad. Boletín de la Asociación de Geógrafos Españoles, 35: 101-122.

ARNÁEZ, J., ORTIGOSA L.M., RUIZ-FLAÑO, P. y LASANTA, T. (2006): Distribución espacial del viñedo en la Comunidad Autónoma de La Rioja: influencia de la topografía y de las formas del relieve. Polígonos. Revista de Geografía, 16: 11-34.

ARNÁEZ, J., RUIZ-FLAÑO, P., LASANTA, T., ORTIGOSA, L., LLORENTE, J.A., PASCUAL, N. y LANA-RENAULT, N. (2012): Efectos de las rodadas de tractores en la escorrentía y erosión de suelos en laderas cultivadas con viñedos. Cuadernos de Investigación Geográfica, 38: 115-130.

BARCO, E. (2008): Análisis de un sector: el Rioja entre dos siglos. Gobierno de La Rioja: 350 pp., Logroño.

BARCO,E., LANGREO, A. y NAVARRO, M.C. (2005): Cambios en el mercado internacional del vino. Distribución y Consumo, 80: 53-64.

BUREL, F. y BAUDRY, J. (2002): Ecología del Paisaje. Conceptos, métodos y aplicaciones. Ediciones Mundi-Prensa: 353 pp., Madrid.

CAÑIZARES RUIZ, M.C. y RUIZ PULPÓN, A.R. (2014): Evolución del paisaje del viñedo en Castilla-La Mancha y revalorización del patrimonio agrario en el contexto de la modernización. Scripta Nova. Revista Electrónica de Geografía y Ciencias Sociales, vol XVIII, no 498. http://www.ub.es/geocrit/sn/sn-498.htm.

CASTELLÓ PUIG, A., FRUTOS MEJÍAS, L.M., HERNÁNDEZ NAVARRO, M.L., LOSCERTALES PALOMAR, B. y RUIZ BUDRÍA, E. (2010): Los paisajes vitivinícolas en la economía rural aragonesa. Actas del XV Coloquio de Geografía Rural: Territorio, paisaje y patrimonio rural. Universidad de Extremadura: 49-61 pp., Cáceres.

CLEERE, H. (2004): World heritage vineyard landscapes. World Heritage Review, 35: 4-19. CONSEJO REGULADOR DE LA DOCaRioja (1991-2013): Memoria anual, Logroño.

CONSTANTINI, E.A. (1992): Study of the relationships between soil suitability for vine cultivation, wine quality and soil erosion through a territorial approach. Geoökoplu, 3: 1-14.

COTS-FOLCH, R., MARTÍNEZ-CASASNOVAS, J.A. y RAMOS, M.C. (2006): Land terracing for new vineyard plantations in the north-eastern Spanish Mediterranean region: Landscape effects of the EU Council Regulation policy for vineyards's restructuring. Agriculture, Ecosystems and Environment, 115: 88-96.

ELÍAS, L.V. (2006): El turismo del vino. Otra experiencia de ocio. Estudios de Ocio, Documento 30. Universidad de Deusto: 256 pp., Bilbao.

ELÍAS, L.V. (2009): Itinerarios del Rioja. Paisaje y bodega en el turismo enológico. En: La Rioja, sus viñas y sus vinos. Gobierno de La Rioja: 257-277, Logroño.

ELÍAS, L.V. (2014): El paisaje del viñedo en La Rioja. Cruce de miradas. Berceo, 167: 39-60.

ESTADISTICA AGRARIA REGIONAL (1987-2013): Memoria anual. Gobierno de La Rioja, Logroño.

FERNÁNDEZ PORTELA, J. (2013): La evolución reciente del sector vitivinícola internacional. Geographos, Vol. 4, nº 39: 173-194. 
FREEMARK, K.E. y MERRIAN, H.G. (1986): Importance of area and habitat heterogeneity to bird assemblages in temperature forest fragments. Biological Conservation, 86: 115141.

GÓMEZ MIGUEL, V. (2006): Mapa de unidades taxonómicas principales de la denominación de origen calificada La Rioja (escala 1:50.000). Atlas Nacional de España: geología, geomorfología y edafología (Sánchez-Ortiz Rodríguez, M. P., coord.). Centro Nacional de Información Geográfica, pp. 195, Madrid.

GRASHOF-BOKDAM, C.J. y VAN LANGEVELDE, F. (2004): Green veining: landscape determinants of biodiversity in European agricultural landscapes. Landscape Ecology, 20: 417-439.

HUETZ DE LEMPS, A. (1967): Vignobles et vins du Nord-Ouest de L'Espagne. Institut de Géographie. Faculté des Lettres, Bordeaux 2 tomos.

JONGMAN, R.H.G. (2002): Homogenisation and fragmentation of the European landscape: ecological consequences and solutions. Landscape and Urban Planning, 58(1-2): 211221.

LASANTA MARTÍNEZ, T. y RUIZ-FLAÑO, P. (2014): Los paisajes del viñedo de Rioja: tradición y modernidad. Berceo, 167: 13-44.

LUGINBÜHL, Y. y VILLA DÍAZ, Á. (2014): Viñas y lagares en las campiñas béticas: un paisaje «fragante»y «resistente». En: Atlas de los paisajes agrarios de España (F. Molinero, coordinador general). Ministerio de Agricultura, Alimentación y Medio Ambiente, Tomo II: 741-748, Madrid.

MAGRAMA. Estadísticas anuales. Gobierno de España. www.magrama.gob.es/

MATA OLMO, R. y FERRER JIMÉNEZ, D. (2014): Viñedos manchegos del Campo de San Juan: la construcción de un paisaje contemporáneo entre la tradición y la modernidad. En: Atlas de los paisajes agrarios de España (F. Molinero, coordinador general). Ministerio de Agricultura, Alimentación y Medio Ambiente, Tomo II: 729-740, Madrid

MARTÍNEZ-CASASNOVAS, J.A., RAMOS, M.C. y COTS-FOLCH, R. (2010): Influence of the EU CAP on terrain morphology and vineyard cultivation in the Priorat region of NE Spain. Land Use Policy, 27: 11-21.

McGARIGAL, K. y MARKS, B. (1995): Fragstats: Spatial pattern analysis program for quantifying landscape structure. Oregon State University.

McGARIGAL, K., CUSHMAN, S.A., NEEL, M.C. y ENE, E. (2002): Fragstats: Spatial Pattern Analysis Program for Categorical Maps. Computer software program produced by the authors at the University of Massachusetts. http://www.umass.edu/landeco/research/ fragstats/fragstats.html

MIRAMONTES CARBALLEDA, A. (2014): El paisaje vitícola en la Galicia mediterránea: complejidad y diversidad de los viñedos de Valdeorras. En: Atlas de los paisajes agrarios de España (F. Molinero, coordinador general). Ministerio de Agricultura, Alimentación y Medio Ambiente, Tomo II: 719-728, Madrid

MOLINERO, F. (2011): Los paisajes del viñedo en Castilla y León: tradición, renovación y consolidación. Polígonos. Revista de Geografía, 21: 85-117.

MOLINERO, F., PORCAL. M.C. y MOLLEVÍ, G. (2013): Viñedos atlánticos, mediterráneos y canarios. En: Atlas de los paisajes agrarios de España (F. Molinero, coordinador general). Ministerio de Agricultura, Alimentación y Medio Ambiente, Tomo I: 193-217, Madrid. 
MOLLEVÍ, G. (2014): El Priorat histórico: la revalorización vitícola. En: Atlas de los paisajes agrarios de España (F. Molinero, coordinador general). Ministerio de Agricultura, Alimentación y Medio Ambiente, Tomo II: 709-718, Madrid.

OIV. Organización Internacional de la Viña y el Vino. http://www.oiv.int/oiv/info/ espresentation?lang=es

PALACIOS GARCÍA, M. (2014): Los barrios de bodegas tradicionales de La Rioja. Berceo, 167: 61-88.

QUIÑONERO-RUBIO, J.M., BOIX-FAYOS, C., DE VENTE, J. (2013): Desarrollo y aplicación de un índice multifactorial de conectividad de sedimentos a escala de Cuenca. Cuadernos de Investigación Geográfica 39 (2), 203-223.

RACLOT, D., LE BISSONNAIS, Y., LOUCHART, X., ANDRIEUX, P., MOUSSA, R. y VOLTZ, M. (2009): Soil tillage and scale effects on erosion from fields to catchment in a Mediterranean vineyard area. Agriculture, Ecosystems and Environment, 134: 201-210.

RAMOS, M.C., COTS-FOLCH, R. y MARTÍNEZ-CASASNOVAS, J.A. (2007): Sustainability of modern land terracing for vineyard plantation in a Mediterranean mountain environment- The case of the Priorat region (NE Spain). Geomorphology, 86: 1-11.

RAMOS, M.C. y MARTÍNEZ-CASASNOVAS, J.A. (2010): Effects of field reorganisation on the spatial variability of runoff and erosion rates in vineyards of Northeastern Spain. Land Degradation \& Development, 21: 1-12.

RICHTER, G., NEGENDANK, J.F.W. (1977): Soil erosion processes and their measurement in German area of Moselle River. Earth Surface Processes and Landforms, 2 (3): 261278.

RUIZ-FLAÑO, P., ARNÁEZ, J., ORTIGOSA, L. y LASANTA, T. (2006): Organización espacial de procesos de erosión en laderas cultivadas con viñedos en La Rioja como consecuencia de precipitaciones intensas. En: Geomorfología y Territorio (A. Pérez Alberti y J. López Bedoya, eds.), Universidade de Santiago de Compostela, Santiago de Compostela, pp. 247-256.

RUIZ PULPÓN, A.R. (2010): Evolución y consolidación del viñedo de regadío en La Mancha. Boletín de la Asociación de Geógrafos Españoles, 52: 5-26.

RUIZ PULPÓN, A.R. (2013): El viñedo en espaldera: nueva realidad en los paisajes vitícolas de Castilla - La Mancha. Boletín de la Asociación de Geógrafos Españoles, 63: 249-270. 
